\title{
The role of submicroscopic malaria in malaria transmission: what is the evidence?
}

\author{
Jessica T. Lin ${ }^{1}$, David L. Saunders ${ }^{2}$, and Steven R. Meshnick ${ }^{3}$ \\ ${ }^{1}$ Division of Infectious Diseases, University of North Carolina School of Medicine, Chapel Hill, NC, \\ USA ${ }^{2}$ Department of Immunology and Medicine, USAMC Armed Forces Research Institute of \\ Medical Sciences, Bangkok, Thailand ${ }^{3}$ Department of Epidemiology, Gillings School of Public \\ Health, University of North Carolina, Chapel Hill, NC, USA
}

\begin{abstract}
Achieving malaria elimination requires targeting the human reservoir of infection, including those with asymptomatic infection. Smear-positive asymptomatic infections detectable by microscopy are an important reservoir because they often persist for months and harbor gametocytes, the parasite stage infectious to mosquitoes. However, many asymptomatic infections are submicroscopic and can only be detected by molecular methods. While there is some evidence that persons with submicroscopic malaria can infect mosquitoes, transmission is much less likely to occur at submicroscopic gametocyte levels. As malaria elimination programs pursue mass screening and treatment of asymptomatic individuals, further research should strive to define the degree to which submicroscopic malaria contributes to the infectious reservoir, and in turn, what diagnostic detection threshold is needed to effectively interrupt transmission.
\end{abstract}

\section{Keywords}

malaria elimination; asymptomatic infection; reservoir; gametocyte; diagnostic

\section{Active surveillance targeting asymptomatic malaria for elimination}

Over the past 5-10 years, increased international funding, political will, and a new generation of malaria diagnostic and treatment tools has reignited efforts to achieve malaria elimination. As of 2012, 34 of the 99 countries where malaria is endemic have adopted strategies to become malaria free within the next two decades [1]. At the heart of these strategies is a shift in focus from early diagnosis and treatment of those who are symptomatic, to active surveillance for and treatment of every case, including those who are asymptomatic $[2,3]$.

\footnotetext{
(C) 2014 Elsevier Ltd. All rights reserved.

Corresponding author: Lin, J.T. (jessica_lin@med.unc.edu).
}

Publisher's Disclaimer: This is a PDF file of an unedited manuscript that has been accepted for publication. As a service to our customers we are providing this early version of the manuscript. The manuscript will undergo copyediting, typesetting, and review of the resulting proof before it is published in its final citable form. Please note that during the production process errors may be discovered which could affect the content, and all legal disclaimers that apply to the journal pertain. 
Asymptomatic malaria is often not defined rigorously, but generally refers to a person harboring blood-stage malaria parasites who has not experienced fever or other symptoms that would lead the individual to seek treatment. In most malaria endemic settings, asymptomatic infections outnumber symptomatic infections $[4,5,6]$. It follows that the success of elimination strategies relies on the ability to find and treat the asymptomatic reservoir.

However, the lack of rigorous definitions for asymptomatic malaria has clouded research supporting this goal $[7,4]$. Parasitemia in an asymptomatic infection can be visible by blood smear, or submicroscopic and only detectable by molecular methods. The increasing use of methods that detect parasite DNA, including PCR and Loop-mediated Isothermal Amplification (LAMP), has uncovered large numbers of asymptomatic persons with submicroscopic malaria. It is generally assumed that this translates into a greatly expanded infectious reservoir. But most studies on the reservoir potential of asymptomatics have been done on microscopically patent asymptomatics. Accordingly, the role of submicroscopic malaria in ongoing transmission in most settings is unclear.

Below, we examine different lines of research to address the question of whether those with submicroscopic malaria contribute substantially to transmission. We highlight the heterogeneity of the asymptomatic malaria reservoir, what mosquito studies have shown about determinants of human-to-mosquito infectivity, the limited studies on the infectivity potential of submicroscopic malaria, and the lack of data available from ongoing elimination efforts.

A better understanding of role of submicroscopic malaria as part of the transmission reservoir can shape how active surveillance is used to pursue malaria elimination. For example, recent mass screening and treatment efforts have used rapid diagnostic tests (RDTs) with detection limits similar to microscopy ( 200 parasites/ $\mu$ l) to find and treat asymptomatic cases [8]. When these efforts failed to curb subsequent malaria transmission, it has been suggested that more sensitive molecular diagnostics need to be deployed $[9,10]$. Along these lines, a recent consensus document by the Malaria Eradication Research Agenda (malERA) consultative group suggests that any parasitemia, no matter how small, represents potential for transmission and therefore is a threat to malaria elimination efforts [11]. We propose that pursuit of the most sensitive diagnostic may be premature. Knowledge gaps are highlighted and recommendations made for further research to better understand how best to deploy active surveillance methods in the pursuit of malaria elimination.

\section{The asymptomatic reservoir is heterogeneous in terms of persistence and gametocyte carriage}

The importance of asymptomatic malaria as a reservoir rests on two key characteristics: duration of infection and gametocyte density. Gametocytes are the sexual stage of the parasite produced in infected human hosts which must be ingested by mosquitoes for transmission to occur. Infections persisting for long periods of time means more opportunities for a malarious person to infect biting mosquitoes, especially since gametocytes tend to appear late in P. falciparum infection, on the order of 7-15 days after

Trends Parasitol. Author manuscript; available in PMC 2015 April 01. 
the first appearance of parasites in the blood [12]. Infections with higher gametocyte densities are generally more infectious. Persistence of infection and prevalent gametocyte carriage have long been observed among those with asymptomatic malaria in moderate to high transmission settings in Africa. However, the asymptomatic reservoir in low transmission settings is more heterogeneous and does not necessarily share these same attributes.

In moderate to high transmission settings in Africa, it is common for asymptomatic individuals to harbor microscopically patent (smear-positive) infections that last from weeks to months $[13,14]$ (Box 1). This phenomenon has been explained by a form of acquired immunity that keeps parasitemia and symptoms in check without achieving complete clearance [15]. Longitudinal genotyping of a Ghanian cohort has confirmed that these are persistent infections of the same strains, rather than representing frequent reinfection, and that they persist an average of 194 days [16]. While gametocytes were not measured in this study, it is likely that these individuals were gametocytemic and thus infectious during much of this time. In fact, asymptomatic microscopically patent infections may be more infectious than clinical malaria. An association between lack of fever and gametocyte carriage has been observed in smear-positive children in the Gambia and Nigeria [17,18]. Additional evidence of the gametocyte production potential of microscopically patent asymptomatics comes from two longitudinal studies in the Gambia and Kenya of untreated asymptomatic infections. $[19,20]$. In these studies, 11-25\% were gametocytemic at baseline, while approximately $15-20 \%$ of microscopically patent asymptomatics without baseline gametocytes became gametocytemic over four weeks. Thus, evidence from a number of studies show that asymptomatic individuals with patent parasitemia are important reservoirs, but none of them looked for submicroscopic infections.

\section{Box 1}

\section{Heterogeneity of the asymptomatic malaria reservoir}

The asymptomatic reservoir is composed of those with submicroscopic and microscopic parasitemia. In both high (Figure IA) and low (Figure IB) transmission settings, asymptomatic infections far outnumber symptomatic infections $[4,5,6]$. However, in low transmission settings, most of the asymptomatic reservoir is composed of submicroscopic parasitemia $[4,21,5,22,23]$. The relative contribution of submicroscopic and microscopic parasitemia to transmission is unknown.

The asymptomatic reservoir's contribution to malaria transmission is mediated by the duration of infection, incidence of gametocyte carriage, and ultimately determined by mosquito infectivity. These and other factors are likely to differ in low vs. high transmission settings and in microscopic vs. submicroscopic parasitemia within these settings (Table I). 


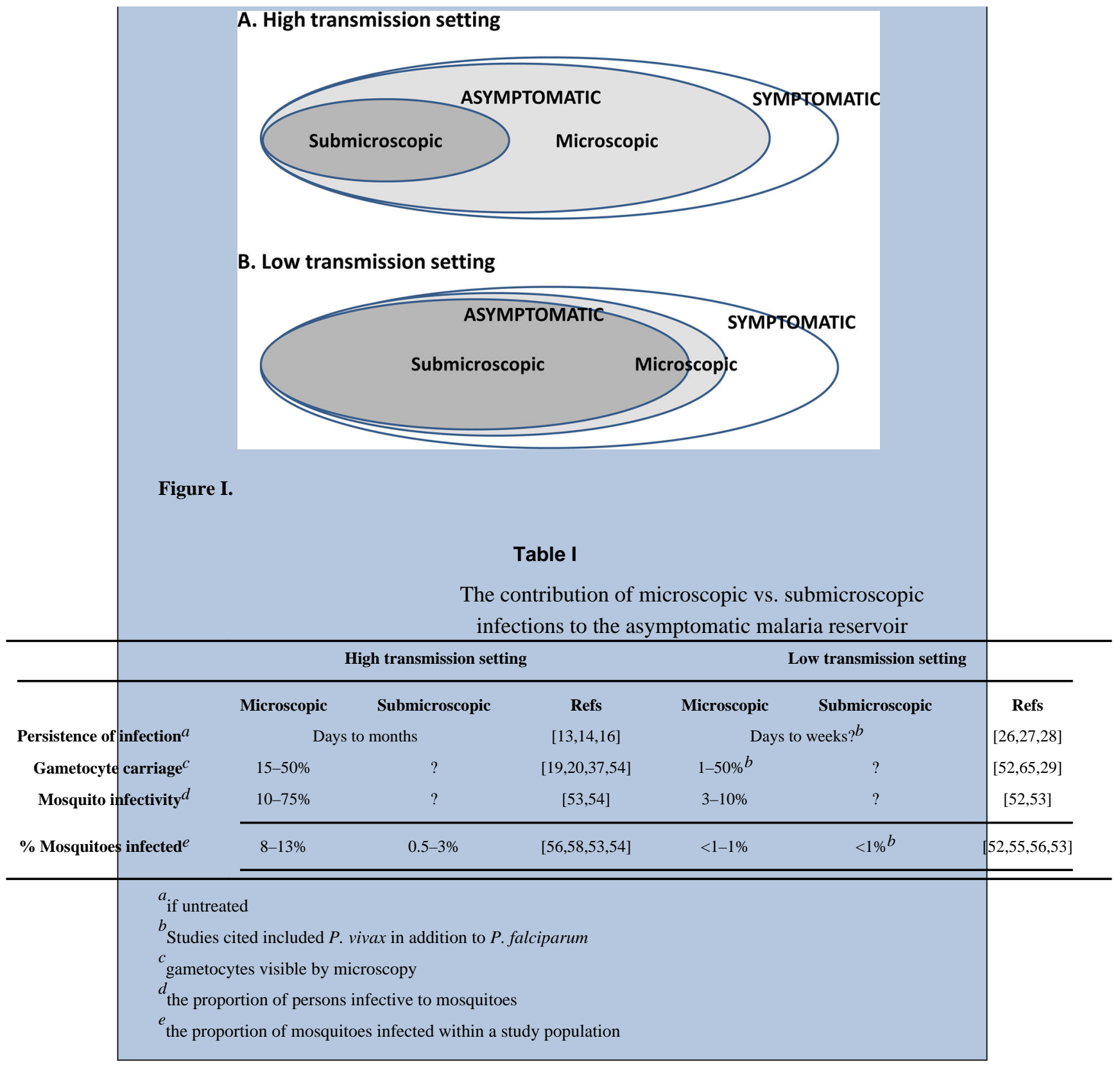

The situation in non-African low transmission settings is more complicated. First, in contrast to many African settings, the majority of asymptomatic malaria infections in areas such as the Amazon and Southeast Asia are submicroscopic [4,21,5,22,23] (Box 1). Second, outside of Africa, Plasmodium vivax is the most prevalent malaria species and malarious areas are often co-endemic for $P$. falciparum and $P$. vivax, with $P$. vivax presenting unique challenges to elimination (Box 2). Finally, with lower levels of transmission, acquired immunity is expected to be lower. So at any given time point, an individual may be more likely to be asymptomatic because he/she is either in the process of resolving parasitemia due to treatment or might be in the process of developing a symptomatic infection [7,4,24,25]. Each of these major differences means that the asymptomatic reservoir may vary in different 
settings with regards to persistence, gametocyte carriage, and hence mosquito infectivity and transmission potential.

\section{Box 2}

\section{$P$. vivax hypnozoites represent an invisible reservoir and obstacle to elimination efforts}

The unique biological characteristics of Plasmodium vivax present even more challenges for elimination [74,1]. Perhaps most important is its propensity to cause relapse. After a $P$. vivax-infected mosquito bites a person, a portion of parasites establish a latent liverstage infection in the form of hypnozoites. Hypnozoites can remain dormant in the liver for weeks or years until unknown triggers cause their reactivation and entry into the bloodstream, where they cause clinical relapse [75]. Remarkably, a single mosquito bite can lead to multiple malaria relapses even when a person has been adequately treated with drugs that clear blood-stage parasites.

Several other biologic characteristics of $P$. vivax make it is plausible that in low transmission settings, many asymptomatic individuals infected with $P$. vivax are gametocytemic and contribute to transmission in a meaningful way. Perhaps due to frequent relapse, most studies show that immunity to $P$. vivax is typically acquired earlier in life than $P$. falciparum in settings where the species are co-endemic [76]. It follows that asymptomatic $P$. vivax infection is relatively common even in low transmission settings [4]. Additionally, in contrast to $P$. falciparum gametocytes, $P$. vivax gametocytes arise early in infection and accompany the majority of symptomatic infections, though they have a relatively short lifespan, on the order of 3 days [32,12]. As with $P$.

falciparum, submicroscopic gametocyte densities have also been shown to lead to mosquito infection [42].

However, the converse conclusion - that mass screening and treatment efforts can interrupt $P$. vivax transmission - is problematic. This is because of the hidden $P$. vivax hypnozoite reservoir. Excluding the pre-patent liver stage of infection, the $P$. falciparum asymptomatic reservoir can be characterized as made up of those who are either smearpositive or submicroscopic, while the $P$. vivax asymptomatic reservoir contains an additional fraction of infected persons without bloodstream parasites but with dormant liver-stage hypnozoites. These persons harboring hypnozoites with potential for relapse cannot be identified by any current diagnostic tests. Thus, one-time or even periodic mass screen-and-treat efforts will only detect infections that have coincidentally reactivated at the time of screening. The size of the hypnozoite reservoir is unknown in all but the most-studied settings, but is likely to be substantial [77]. Perhaps this is why the few examples of successful interruption of $P$. vivax transmission have been associated with Mass Drug Administration (MDA) [78,79] rather than screen and treatment efforts.

\section{Research recommendations}

Ultrasensitive molecular tests might incrementally detect a larger proportion of submicroscopic and potentially gametocytemic $P$. vivax infections within a population, but their use is perhaps inconsequential for achieving elimination in the face of a much 
larger hidden hypnozoite reservoir that remains intact. Lines of research that evaluate frequent periodic screening, replace mass screening with MDA with the aid of point-ofcare G6PD testing, or uncover triggers of relapse can inform active surveillance strategies for $P$. vivax. However, for most areas where $P$. vivax is endemic, development of a diagnostic test that can detect latent hypnozoite infections in conjunction with a safe single-dose anti-hypnozoite drug and are probably needed in order to make elimination a realistic goal $[11,80,74]$.

The duration of infection among asymptomatics in low transmission settings remains elusive (Box 1). The very limited evidence suggests that a minority can persist for several weeks, but the majority spontaneously clear. In Brazil, 16 submicroscopic PCR-positive persons with asymptomatic Plasmodium vivax infection were followed for 30 days; three-quarters were PCR-negative at 2 weeks, while the remaining four remained PCR-positive at 30 days, with none developing symptoms [26]. In serial cross-sectional studies conducted in Colombia and Peru, roughly half of smear-positive asymptomatic infections spontaneously cleared within 14 days in Colombia (13/24) and within 7 days in Peru (16/30) [27,28,29]. As for the rest, the majority remained asymptomatic at follow-up, with half of those in the Peru study showing decreased parasitemia at day 7 , and $25 \%$ of those in the Colombian study remaining parasitemic at day 14 . Overall, the duration of untreated submicroscopic infections in low-transmission areas seems to be much shorter than asymptomatic $P$. falciparum infections in Africa.

Even less is known about gametocyte carriage among asymptomatic infections in low transmission settings. One study in Peru limited to smear-positive malaria found that an impressive $50 \%$ of $P$. falciparum and $22 \% P$. vivax infections identified by active case detection harbored gametocytes [29]. However this was in the context of an overall malaria prevalence of $63 \%$ in the at-risk zones that were targeted. Otherwise, studies of gametocyte carriage have mostly been limited to those enrolled in clinical trials. Studies in Thailand have found that the greatest risk factor for $P$. falciparum gametocyte carriage was visible gametocytemia at admission [30], present in just $2.4 \%$ of patients. Meanwhile, the average duration of gametocytemia post-treatment in Thailand is significantly shorter than in the Gambia [31]. For P. vivax patients in Thailand and Indonesia, gametocyte carriage mirrored asexual parasitemia, with high asexual parasitemia associated with increased risk of gametocytemia during follow-up [32]. These studies perhaps indirectly suggest that submicroscopic asymptomatic malaria does not have the same infectious potential as asymptomatic malaria in Africa.

\section{Studies of mosquito infectivity indicate a gametocyte threshold detectable by microscopy}

Gametocytes serve as an imperfect surrogate marker of transmission potential. Experimental studies whereby mosquitoes are fed blood-stage parasites, either by direct skin feeding or via a membrane feeding apparatus, and subsequently dissected to look for malaria parasites (oocysts in the mosquito midgut), represent a more direct way for assessing human-tomosquito infectious potential $[33,34,35,36]$. 
Individuals with higher gametocytemia are generally more infectious to mosquitoes than those with low gametocytemia. While children in Africa without smear-detectable gametocytes are capable of infecting mosquitoes, infections occur in only a small minority of mosquitoes [37,38]. In contrast, evidence from many studies suggests a threshold of $\sim 100$ gametocytes $/ \mu \mathrm{L}$, above which individual more consistently achieved infection in the majority of mosquitoes, and cause mosquito infections with higher oocyst burdens[39,40] (Figure 2). More recent data based on modeling of mosquito membrane feeding studies in Burkina Faso and Kenya similarly found that while $4 \%$ of mosquitoes are infected at 1 gametocyte/ $\mu \mathrm{L}$, this low infectivity rate does not increase meaningfully until $\sim 200$ gametocytes $/ \mu \mathrm{L}$ are present [41] (Figure 2).

Comparable studies for $P$. vivax are few in number. While they suggest a positive correlation between gametocyte density and frequency of mosquito infection, an appreciable gametocyte threshold above which infection is likely has not been defined $[35,42,43]$.

Because the density of gametocytes relative to asexual parasites in $P$. falciparum infection is usually low fewer than 5\% in most settings [44] - a gametocyte threshold of 100 gametocytes $/ \mu \mathrm{L}$ roughly translates into an overall parasitemia of 2000 parasites $/ \mu \mathrm{L}$, well into the range of parasitemia detectable by microscopy. There may be parasite and host factors beyond gametocyte density that are important determinants of human-to-mosquito infectivity $[12,45,46,47]$, and membrane feeding assays may still be an imperfect way of measuring human infectiousness to mosquitoes [48). Overall, these data argue that submicroscopic infections may not contribute substantially to the reservoir.

\section{The potential role of submicroscopic malaria in transmission has only been indirectly studied in high transmission settings with prevalent gametocyte carriage}

Should all parasitemic individuals be considered current or future gametocytes producers and, by implication, treated $[12,49]$ ? Studies supporting this rationale have been largely confined to children in Africa [37,38], a group where gametocyte carriage is expected to be more prevalent. Further, while microscopically positive gametocytemic individuals are clearly infectious to mosquitoes, some studies highlighting the infectious potential of submicroscopic gametocytemia can be hard to interpret because they do not specify whether such subjects also had submicroscopic malaria (i.e., they could have had detectable asexual stages).

Among a random sample of 80 children in Burkina Faso, roughly twice as many of those with microscopically detectable gametocytes were infectious to mosquitoes compared to those with gametocytes detectable only by molecular methods: $68 \%$ with smear-detectable gametocytes infected on average $13 \%$ of mosquitoes, while $32 \%$ with submicroscopic gametocytes infected on average $2.3 \%$ of mosquitoes [37]. Based on these numbers, it was estimated that those with submicroscopic gametocytes contributed to $24.2 \%$ of overall transmission because of their greater prevalence in the population. However, $<20 \%$ of children in this study had submicroscopic malaria, so the majority with submicroscopic 
gametocytemia likely still had asexual parasites visible by smear. Another study in western Kenya also found that submicroscopic gametocytemia frequently resulted in mosquito infection [38]. However, the study recruited children who were 14 days post-treatment, mostly with SP-containing regimens, which have been associated with increased posttreatment gametocytemia $[50,51,31]$.

Human-to-mosquito infectivity studies among asymptomatics in Thailand also found persons harboring submicroscopic gametocytemia that were infectious to mosquitoes, but these were among microscopically patent infections. Village-wide screening by microscopy in western Thailand resulted in 3.3\% of persons infected with P. falciparum by microscopy, $80 \%$ of whom were asymptomatic [52]. Among the 51 infected persons in whom gametocytes were not visible, 5 (9.8\%) still infected mosquitoes, yielding low oocyst burdens (average 1.2 oocysts/mosquito). Meanwhile, $13.5 \%$ of the $P$. vivax patients without smear detectable gametocytes also infected mosquitoes. Overall, $10 \%$ of identified $P$. falciparum infections transmitted their infection to mosquitoes in a membrane-feeding assay, a rate similar to that seen in asymptomatics found by active surveillance in two villages in Cameroon (9-13\%) [53], but much lower than that observed in a village sample in Burkina Faso ( $>50 \%)$ and treated children in western Kenya $(62 \%)[54,38]$. These studies suggest that the underlying gametocyte prevalence in different settings can vary widely and has an impact on the size of the infectious reservoir [12,31].

Should PCR be used to screen for the transmission reservoir? Epidemiologic data pertaining directly to this question are scarce. In one study in the Brazilian Amazon, 15 asymptomatic carriers (11 P. vivax, 3 P. falciparum, 1 mixed P. falciparum/vivax) detected in a mass survey who were microscopy-negative but PCR positive were recruited for mosquito feeding experiments two months after PCR diagnosis. Of 294 mosquitoes fed on their blood by both direct and membrane feeding, two mosquitoes became infected with one $P$. vivax oocyst each, yielding an infection rate of $0.7 \%$ [55]. Comparing this to a mosquito infection rate of $22 \%$ among 17 symptomatic carriers with microscopically detectable parasitemia in the same study, yields an approximately 300 fold difference in infectivity between submicroscopic asymptomatics and microscopic symptomatics in this setting.

Further epidemiologic data gleaned from three human-to-mosquito infectivity studies was recently cited to compare mosquito infectivity between submicroscopic and microscopic asymptomatics. These showed between a 4 and 16-fold difference in $\%$ mosquitoes infected among submicroscopic vs. slide-positive P. falciparum infections [56]. However, one of these studies involved African-American malariotherapy patients with neurosyphilis who were partially treated [57], another represents unpublished data from Burkina Faso, and in the third study from South Carolina in 1948 [58], the prevalence of submicroscopic carriers had to be estimated. All three studies appear to be drawn from populations where the underlying gametocyte prevalence is high: slide detectable gametocyte prevalence was $\checkmark 68 \%$ in the malariaotherapy study and 64\% in the South Carolina study [57,58].

Gametocyte prevalence in areas where malaria is now hypoendemic is expected to be much lower. Thus, these 4 to 16 -fold infectivity ratios are only rough estimates. These estimates were used to suggest that submicroscopic carriers are the source of 20-50\% of all human-tomosquito transmissions when transmission reaches very low levels [56]. This conclusion, 
while based on the best available evidence, underscores the need for more epidemiologic data, especially from low transmission settings where malaria elimination efforts are underway.

\section{Sparse data exist from elimination efforts using active surveillance to guide practice}

As countries pursue malaria elimination, interventions ranging from focal or mass screening and treatment to mass drug administration to targeted populations are being tried [3]. Reactive case detection, whereby households within an established radius surrounding a symptomatic positive index case are screened, is also increasingly being used as a strategy that bridges passive and active surveillance [59,60,61]. Many of these efforts are applying molecular methods to find and treat asymptomatic infections [23,62,63,64]. But we found only two attempts to measure gametocytes in such studies. In a school and population-based survey in the Melanesian Islands where an overall malaria point prevalence of 2-3\% was found by both microscopy and PCR, gametocytes were detected in $15 \%$ of the P. falciparum positives and $4 \%$ of the $P$. vivax positives [65]. In northwest Thailand, PCR of 475 persons in two villages as part of a mass screening revealed eight cases with low density parasitemia later confirmed by expert microscopists. None of these carried gametocytes visible by smear, but submicroscopic gametocytes were not measured [66]. Because infected persons are treated, the duration of these submicroscopic cases is also unknown. The possibility exists that the submicroscopic infections found by such surveillance efforts might not contribute significantly to the transmission reservoir [3].

\section{Research strategies}

Because of the logistical difficulties of studying asymptomatic infections, much of what is known about asymptomatic malaria in African settings has been extrapolated to settings with much lower transmission where the asymptomatic reservoir mostly consists of those with submicroscopic malaria. Studies of duration of infection, submicroscopic gametocyte carriage, and gametocyte production potential need to be carried out in submicroscopic infections in low transmission settings where elimination is being considered (Box 3). This can be done, either by retrospective molecular analysis of prior cohort studies where only microscopy was used, or prospectively, in the context of cohort studies and national surveys, in which microscopy-negative but PCR-positive subjects are followed and not treated unless they become symptomatic or develop patent parasitemia. These studies are made more feasible by available methods to measure submicroscopic gametocytemia from blood spots collected for PCR $[67,68,69,70,71]$ but require longitudinal follow up which can be challenging.

\section{Box 3}

\section{Outstanding questions and possible strategies to answer them}

\begin{tabular}{|l|l|}
\hline Research Question & Possible Study Designs \\
\hline Submicroscopic parasitemia &
\end{tabular}

Submicroscopic parasitemia 


\begin{tabular}{|c|c|}
\hline Research Question & Possible Study Designs \\
\hline $\begin{array}{l}\text { What proportion of untreated/unrecognized } \\
\text { submicroscopic infections persist asymptomatically? }\end{array}$ & \multirow[t]{2}{*}{$\begin{array}{l}\text { Retrospective molecular analysis of prior cohort } \\
\text { studies where only microscopy was used. }\end{array}$} \\
\hline $\begin{array}{l}\text { What proportion go on to become microscopically } \\
\text { patent and/or symptomatic? }\end{array}$ & \\
\hline $\begin{array}{l}\text { How often do treated patients develop subsequent } \\
\text { submicroscopic parasitemia? }\end{array}$ & $\begin{array}{l}\text { Retrospective molecular analysis of therapeutic } \\
\text { efficacy studies. }\end{array}$ \\
\hline \multicolumn{2}{|l|}{ Transmission potential of submicroscopic infections } \\
\hline $\begin{array}{l}\text { What proportion of submicroscopic infections } \\
\text { harbor or produce gametocytes, and at what density? }\end{array}$ & $\begin{array}{l}\text { Prospective molecular analysis incorporated into } \\
\text { cohort studies or national surveys. }\end{array}$ \\
\hline $\begin{array}{l}\text { Besides gametocyte density, what parasite or } \\
\text { human host factors affect human-to-mosquito } \\
\text { infectivity? }\end{array}$ & In vitro membrane feeding assays \\
\hline $\begin{array}{l}\text { How commonly does submicroscopic vs. } \\
\text { microscopic malaria in asymptomatics successfully } \\
\text { infect mosquitoes? How does this vary in different } \\
\text { transmission settings and in different mosquito } \\
\text { vectors? }\end{array}$ & $\begin{array}{l}\text { Vectorial capacity studies, estimates of } \\
\text { Entomological Inoculation Rate (EIR), mosquito } \\
\text { membrane feeding studies in vitro and from } \\
\text { patients in surveys and/or cohorts }\end{array}$ \\
\hline \multicolumn{2}{|c|}{ Diagnostic tests to detect submicroscopic parasitemia and estimate transmissibility } \\
\hline $\begin{array}{l}\text { How sensitive does a diagnostic test need to be to } \\
\text { identify all those in the infectious reservoir? }\end{array}$ & $\begin{array}{l}\text { Comparative analysis of diagnostic tests } \\
\text { incorporated into cohort studies and mass surveys, } \\
\text { paired with malaria seroprevalence surveys }\end{array}$ \\
\hline $\begin{array}{l}\text { How large is the hypnozoite reservoir in } P \text {. vivax } \\
\text { endemic areas, and how can it be measured? }\end{array}$ & $\begin{array}{l}\text { Studies to identify surrogate markers of latent } P \text {. } \\
\text { vivax infection }\end{array}$ \\
\hline
\end{tabular}

Going one step further, human-to-mosquito infectivity studies can provide more direct information about the transmission potential of submicroscopic infections (Box 3). Ideally, serial mosquito membrane feeding studies using local mosquito vectors would be conducted in those with asymptomatic submicroscopic malaria identified by current mass screening and treatment efforts. While challenging and tedious, these would more directly measure the potential impact that submicroscopic cases uncovered by active surveillance have on transmission. Since such studies still do not measure mosquito-to-human transmission or overall malaria incidence, cluster randomized trials of different interventions, for example mass screening and treatment vs. mass drug administration vs. vector control, remain important [72]. Incorporating different types of molecular diagnostics (RDT, PCR, and LAMP) into such studies would allow better definition of a diagnostic threshold for elimination below which transmission is unlikely, a practical goal that could guide allocation of resources for diagnostics in malaria elimination programs.

The policy implications are important: if individuals found by active surveillance to have submicroscopic malaria turn out to have minimal transmission potential, perhaps ultrasensitive PCR methods are not as important as an emphasis on better coverage and follow up of targeted populations. If partially treated or pre-symptomatic persons make up a significant part of the asymptomatic reservoir, intensifying malaria treatment efforts to involve periodic follow up post-treatment as well as enhancing early detection might be more cost-effective than screening large populations. 


\section{Concluding remarks}

As resources are allotted to elimination efforts, further research is critical to better characterize the asymptomatic reservoir in settings where elimination is being pursued and the factors that may or may not make submicroscopic infections important for continuing transmission. In particular, a key operational question is, "how sensitive does a diagnostic test need to be to identify all of those who make up the infectious reservoir?" Because a gametocyte-mosquito infectivity threshold has been established for $P$. falciparum in African settings, this can act as a guide. Nevertheless, determining the gametocyte carriage potential of individuals followed without treatment is also key, especially for those with submicroscopic parasitemia. In regards to infection with $P$. vivax, the present inventory of tools is inadequate to achieve elimination without a diagnostic marker for latent hypnozoite infection or introduction of a universally safe antimalarial that can clear hypnozoites.

Presently, the lack of evidence for or against a substantive role of submicroscopic malaria for transmission makes the ethics of treating asymptomatic and submicroscopic parasitemia unclear and dependent on the risk-benefit ratios of the treatment and/or prevention strategies being implemented. Untreated individuals may go on to develop clinical disease at rates that are currently unpredictable and infect others in their households or communities.

Alternatively, infection could clear despite treatment that might cause adverse effects in patients. If the treatment is ineffective, they may continue to harbor persistent, increasingly drug-resistant parasites post treatment. The spread of infection, while thought by some to be from a widespread reservoir of asymptomatic individuals, may in fact be limited to only a relatively small pool of patients with microscopically patent infection. The sporadic and focal nature of malaria transmission argues for the latter hypothesis. However, even if transmission is focal, and limited to a few carriers, the potential beneficial effects of mass treatment to provide prophylaxis and disrupt transmission must be considered carefully [73]. Longitudinal studies that incorporate molecular analyses and mosquito membrane feeding techniques will help tease out some of these outcomes and contribute substantially to our understanding of how best to target the asymptomatic malaria reservoir.

\section{Acknowledgments}

This work was supported by an American Society of Tropical Medicine \& Hygiene/Burroughs Wellcome Postdoctoral Fellowship in Tropical Infectious Disease to J.T.L., the U.S. Department of Defense Global Emerging Infections Surveillance and Response System Program, and National Institute of Allergy and Infectious Diseases (NIH grant R56AI097609 to S.R.M). The views expressed in this paper are those of the authors and do not represent the official position of the U.S. Department of Defense.

\section{References}

1. Cotter $\mathrm{C}$, et al. The changing epidemiology of malaria elimination: new strategies for new challenges. Lancet. 2013; 382:900-911. [PubMed: 23594387]

2. Mendis K, et al. From malaria control to eradication: The WHO perspective. Trop Med Int Health. 2009; 14:802-809. [PubMed: 19497083]

3. Sturrock HJW, et al. Targeting asymptomatic malaria infections: active surveillance in control and elimination. PLoS Med. 2013; 10:e1001467. [PubMed: 23853551]

4. Lindblade KA, et al. The silent threat: asymptomatic parasitemia and malaria transmission. Expert Rev Anti Infect Ther. 2013; 11:623-639. [PubMed: 23750733] 
5. Alves FP, et al. High prevalence of asymptomatic Plasmodium vivax and Plasmodium falciparum infections in native Amazonian populations. Am J Trop Med Hyg. 2002; 66:641-648. [PubMed: 12224567]

6. Pinto J, et al. Malaria in São Tomé and Príncipe: parasite prevalences and vector densities. Acta Trop. 2000; 76:185-193. [PubMed: 10936578]

7. Laishram DD, et al. The complexities of malaria disease manifestations with a focus on asymptomatic malaria. Malar J. 2012; 11:29. [PubMed: 22289302]

8. Sutcliffe CG, et al. Reduced risk of malaria parasitemia following household screening and treatment: a cross-sectional and longitudinal cohort study. PLoS ONE. 2012; 7:e31396. [PubMed: 22319629]

9. Tiono AB, et al. A controlled, parallel, cluster-randomized trial of community-wide screening and treatment of asymptomatic carriers of Plasmodium falciparum in Burkina Faso. Malar J. 2013; 12:79. [PubMed: 23442748]

10. Hoyer S, et al. Focused Screening and Treatment (FSAT): a PCR-based strategy to detect malaria parasite carriers and contain drug resistant $P$. falciparum, Pailin, Cambodia. PLoS ONE. 2012; 7:e45797. [PubMed: 23049687]

11. malERA Consultative Group on Diagnoses and Diagnostics. A research agenda for malaria eradication: diagnoses and diagnostics. PLoS Med. 2011; 8:e1000396. [PubMed: 21311583]

12. Bousema T, Drakeley C. Epidemiology and infectivity of Plasmodium falciparum and Plasmodium vivax gametocytes in relation to malaria control and elimination. Clin Microbiol Rev. 2011; 24:377-410. [PubMed: 21482730]

13. Males S, et al. Long-term asymptomatic carriage of Plasmodium falciparum protects from malaria attacks: a prospective study among Senegalese children. Clin Infect Dis. 2008; 46:516-522. [PubMed: 18199040]

14. Bottius E, et al. Malaria: even more chronic in nature than previously thought; evidence for subpatent parasitaemia detectable by the polymerase chain reaction. Trans R Soc Trop Med Hyg. 1996; 90:15-19. [PubMed: 8730301]

15. Doolan DL, et al. Acquired immunity to malaria. Clin Microbiol Rev. 2009; 22:13-36. [PubMed: 19136431]

16. Felger I, et al. The dynamics of natural Plasmodium falciparum infections. PLoS ONE. 2012; 7:e45542. [PubMed: 23029082]

17. Von Seidlein L, et al. Risk factors for gametocyte carriage in Gambian children. Am J Trop Med Hyg. 2001; 65:523-527. [PubMed: 11716108]

18. Sowunmi A, et al. Risk factors for gametocyte carriage in uncomplicated falciparum malaria in children. Parasitology. 2004; 129:255-262. [PubMed: 15471001]

19. Dunyo S, et al. Gametocytaemia after drug treatment of asymptomatic Plasmodium falciparum. PLoS Clin Trials. 2006; 1:e20. [PubMed: 17013431]

20. Bousema JT, et al. Plasmodium falciparum gametocyte carriage in asymptomatic children in western Kenya. Malar J. 2004; 3:18. [PubMed: 15202944]

21. Okell LC, et al. Submicroscopic infection in Plasmodium falciparum-endemic populations: a systematic review and meta-analysis. J Infect Dis. 2009; 200:1509-1517. [PubMed: 19848588]

22. Steenkeste N, et al. Sub-microscopic malaria cases and mixed malaria infection in a remote area of high malaria endemicity in Rattanakiri province, Cambodia: implication for malaria elimination. Malar J. 2010; 9:108. [PubMed: 20409349]

23. Harris I, et al. A large proportion of asymptomatic Plasmodium infections with low and submicroscopic parasite densities in the low transmission setting of Temotu Province, Solomon Islands: challenges for malaria diagnostics in an elimination setting. Malar J. 2010; 9:254. [PubMed: 20822506]

24. El-Sayed B, et al. A randomized open-label trial of artesunate- sulfadoxine-pyrimethamine with or without primaquine for elimination of sub-microscopic $P$. falciparum parasitaemia and gametocyte carriage in eastern Sudan. PLoS ONE. 2007; 2:e1311. [PubMed: 18074034]

25. da Silva-Nunes M, Ferreira MU. Clinical spectrum of uncomplicated malaria in semi-immune Amazonians: beyond the 'symptomatic' vs 'asymptomatic' dichotomy. Memórias do Instituto Oswaldo Cruz. 2007; 102:341-348. [PubMed: 17568940] 
26. Camargo EP, et al. Symptomless Plasmodium vivax infections in native Amazonians. Lancet. 1999; 353:1415-1416. [PubMed: 10227233]

27. Cucunubá ZM, et al. Asymptomatic Plasmodium spp. infection in Tierralta, Colombia. Mem Inst Oswaldo Cruz. 2008; 103:668-673. [PubMed: 19057816]

28. Cucunubá ZM, et al. Comparison of asymptomatic Plasmodium spp. infection in two malariaendemic Colombian locations. Trans R Soc Trop Med Hyg. 2013; 107:129-136. [PubMed: 23222954]

29. Branch O, et al. Clustered local transmission and asymptomatic Plasmodium falciparum and Plasmodium vivax malaria infections in a recently emerged, hypoendemic Peruvian Amazon community. Malaria Journal. 2005; 4:27. [PubMed: 15975146]

30. Price R, et al. Risk factors for gametocyte carriage in uncomplicated falciparum malaria. Am J Trop Med Hyg. 1999; 60:1019-1023. [PubMed: 10403336]

31. Stepniewska K, et al. Plasmodium falciparum gametocyte dynamics in areas of different malaria endemicity. Malar J. 2008; 7:249. [PubMed: 19055751]

32. Douglas NM, et al. Gametocyte Dynamics and the Role of Drugs in Reducing the Transmission Potential of Plasmodium vivax. J Infect Dis. 2013; 208:801-812. [PubMed: 23766527]

33. Bousema T, et al. Mosquito feeding assays to determine the infectiousness of naturally infected Plasmodium falciparum gametocyte carriers. PLoS ONE. 2012; 7:e42821. (2012). [PubMed: 22936993]

34. Stone WJR, et al. The relevance and applicability of oocyst prevalence as a read-out for mosquito feeding assays. Sci Rep. 2013; 3:3418. [PubMed: 24301557]

35. Sattabongkot J, et al. Comparison of artificial membrane feeding with direct skin feeding to estimate the infectiousness of Plasmodium vivax gametocyte carriers to mosquitoes. Am J Trop Med Hyg. 2003; 69:529-535. [PubMed: 14695091]

36. Gamage-Mendis AC, et al. Infectivity of Plasmodium vivax and P. falciparum to Anopheles tessellatus; relationship between oocyst and sporozoite development. Trans R Soc Trop Med Hyg. 1993; 87:3-6. [PubMed: 8465388]

37. Ouédraogo AL, et al. Substantial contribution of submicroscopical Plasmodium falciparum gametocyte carriage to the infectious reservoir in an area of seasonal transmission. PLoS ONE. 2009; 4:e8410. [PubMed: 20027314]

38. Schneider P, et al. Submicroscopic Plasmodium falciparum gametocyte densities frequently result in mosquito infection. Am J Trop Med Hyg. 2007; 76:470-474. [PubMed: 17360869]

39. Carter, R.; Graves, PM. Gametocytes. In: Wernsorfer, WH.; McGregor, I., editors. Malaria: Principles and Practice of Malariology. Churchill Livingstone; 1988. p. 253-305.

40. Bell AS, et al. Enhanced Transmission of Drug-Resistant Parasites to Mosquitoes following Drug Treatment in Rodent Malaria. PLoS ONE. 2012; 7:e37172. [PubMed: 22701563]

41. Churcher TS, et al. Predicting mosquito infection from Plasmodium falciparum gametocyte density and estimating the reservoir of infection. Elife. 2013; 2:e00626. [PubMed: 23705071]

42. Bharti AR, et al. Experimental infection of the neotropical malaria vector Anopheles darlingi by human patient-derived Plasmodium vivax in the Peruvian Amazon. Am J Trop Med Hyg. 2006; 75:610-616. [PubMed: 17038681]

43. Sattabongkot J, et al. Plasmodium vivax: gametocyte infectivity of naturally infected Thai adults. Parasitology. 1991; 102(Pt 1):27-31. [PubMed: 2038501]

44. Taylor LH, Read AF. Why so few transmission stages? Reproductive restraint by malaria parasites. Parasitol Today. 1997; 13:135-140. [PubMed: 15275099]

45. Nwakanma D, et al. High gametocyte complexity and mosquito infectivity of Plasmodium falciparum in the Gambia. Int J Parasitol. 2008; 38:219-227. [PubMed: 17709108]

46. Paul REL, et al. Aggregation in malaria parasites places limits on mosquito infection rates. Infect Genet Evol. 2007; 7:577-586. [PubMed: 17521970]

47. McQueen PG, et al. Host immune constraints on malaria transmission: insights from population biology of within-host parasites. Malar J. 2013; 12:206. [PubMed: 23767770] 
48. Gouagna LC, et al. Comparison of field-based xenodiagnosis and direct membrane feeding assays for evaluating host infectiousness to malaria vector Anopheles gambiae. Acta Trop. 2013; 130C: 131-139. [PubMed: 24262642]

49. Mosha JF, et al. Epidemiology of subpatent Plasmodium falciparum infection: implications for detection of hotspots with imperfect diagnostics. Malar J. 2013; 12:221. [PubMed: 23815811]

50. Barnes KI, et al. Increased gametocytemia after treatment: an early parasitological indicator of emerging sulfadoxine-pyrimethamine resistance in falciparum malaria. J Infect Dis. 2008; 197:1605-1613. [PubMed: 18471066]

51. Bousema JT, et al. Moderate effect of artemisinin-based combination therapy on transmission of Plasmodium falciparum. J Infect Dis. 2006; 193:1151-1159. [PubMed: 16544256]

52. Coleman RE, et al. Infectivity of asymptomatic Plasmodium-infected human populations to Anopheles dirus mosquitoes in western Thailand. J Med Entomol. 2004; 41:201-208. [PubMed: 15061279]

53. Bonnet $\mathrm{S}$, et al. Estimation of malaria transmission from humans to mosquitoes in two neighbouring villages in south Cameroon: evaluation and comparison of several indices. Trans $\mathrm{R}$ Soc Trop Med Hyg. 2003; 97:53-59. [PubMed: 12886806]

54. Boudin C, et al. High human malarial infectivity to laboratory-bred Anopheles gambiae in a village in Burkina Faso. Am J Trop Med Hyg. 1993; 48:700-706. [PubMed: 8517489]

55. Alves FP, et al. Asymptomatic carriers of Plasmodium spp. as infection source for malaria vector mosquitoes in the Brazilian Amazon. J Med Entomol. 2005; 42:777-779. [PubMed: 16363160]

56. Okell LC, et al. Factors determining the occurrence of submicroscopic malaria infections and their relevance for control. Nat Commun. 2012; 3:1237. [PubMed: 23212366]

57. Jeffert GM, Eyles DE. Infectivity to mosquitoes of Plasmodium falciparum as related to gametocyte density and duration of infection. Am J Trop Med Hyg. 1955; 4:781-789. [PubMed: 13259002]

58. Young MD, Hardman NF. The infectivity of native malarias in South Carolina to Anopheles quadrimaculatus. Am J Trop Med Hyg. 1948; 28:303-311. [PubMed: 18858032]

59. Rogawski ET, et al. Active case detection with pooled real-time PCR to eliminate malaria in Trat province, Thailand. Am J Trop Med Hyg. 2012; 86:789-791. [PubMed: 22556075]

60. Sturrock HJW, et al. Reactive case detection for malaria elimination: real-life experience from an ongoing program in Swaziland. PLoS ONE. 2013; 8:e63830. [PubMed: 23700437]

61. Littrell M, et al. Case investigation and reactive case detection for malaria elimination in northern Senegal. Malar J. 2013; 12:331. [PubMed: 24044506]

62. Kritsiriwuthinan K, Ngrenngarmlert W. Molecular screening of Plasmodium infections among migrant workers in Thailand. J Vector Borne Dis. 2011; 48:214-218. [PubMed: 22297283]

63. De AW, Gunasekera WMKT, et al. Usefulness of polymerase chain reaction to supplement field microscopy in a pre-selected population with a high probability of malaria infections. Am J Trop Med Hyg. 2011; 85:6-11. [PubMed: 21734117]

64. Atkinson JA, et al. Operational research to inform a sub-national surveillance intervention for malaria elimination in Solomon Islands. Malar J. 2012; 11:101. [PubMed: 22462770]

65. Pacific Malaria Initiative Survey Group (PMISG) on behalf of the Ministries of Health of Vanuatu and Solomon Islands. Malaria on isolated Melanesian islands prior to the initiation of malaria elimination activities. Malar J. 2010; 9:218. [PubMed: 20659316]

66. Congpuong K, et al. Mass blood survey for malaria: pooling and real-time PCR combined with expert microscopy in north-west Thailand. Malar J. 2012; 11:288. [PubMed: 22909399]

67. Babiker HA, Schneider P. Application of molecular methods for monitoring transmission stages of malaria parasites. Biomed Mater. 2008; 3:034007. [PubMed: 18708712]

68. Mlambo G, et al. A filter paper method for the detection of Plasmodium falciparum gametocytes by reverse transcription polymerase chain reaction. Am J Trop Med Hyg. 2008; 78:114-116. [PubMed: 18187793]

69. Schneider P, et al. Quantification of Plasmodium falciparum gametocytes in differential stages of development by quantitative nucleic acid sequence-based amplification. Mol Biochem Parasitol. 2004; 137:35-41. [PubMed: 15279949] 
70. Buates S, et al. Development of a reverse transcription-loop-mediated isothermal amplification (RT-LAMP) for clinical detection of Plasmodium falciparum gametocytes. Parasitol Int. 2010; 59:414-420. [PubMed: 20542143]

71. Wampfler R, et al. Strategies for detection of Plasmodium species gametocytes. PLoS ONE. 2013; 8:e76316. [PubMed: 24312682]

72. Tiono AB, et al. Dynamics of malaria transmission and susceptibility to clinical malaria episodes following treatment of Plasmodium falciparum asymptomatic carriers: results of a clusterrandomized study of community-wide screening and treatment, and a parallel entomology study. BMC Infect Dis. 2013; 13:535. [PubMed: 24215306]

73. Okell LC, et al. The potential contribution of mass treatment to the control of Plasmodium falciparum malaria. PLoS ONE. 2011; 6:e20179. [PubMed: 21629651]

74. WHO. Global malaria control and elimination: report of a technical review. WHO; 2008. at <http:// www.who.int/malaria/publications/atoz/9789241596756/en/>

75. White NJ. Determinants of relapse periodicity in Plasmodium vivax malaria. Malaria Journal. 2011; 10:297. [PubMed: 21989376]

76. Mueller I, et al. Natural acquisition of immunity to Plasmodium vivax: epidemiological observations and potential targets. Adv Parasitol. 2013; 81:77-131. [PubMed: 23384622]

77. Betuela I, et al. Relapses contribute significantly to the risk of Plasmodium vivax infection and disease in Papua New Guinean children 1-5 years of age. J Infect Dis. 2012; 206:1771-1780. [PubMed: 22966124]

78. Kaneko A, et al. Malaria eradication on islands. Lancet. 2000; 356:1560-1564. [PubMed: 11075770]

79. Hsiang MS, et al. Mass drug administration for the control and elimination of Plasmodium vivax malaria: an ecological study from Jiangsu province, China. Malar J. 2013; 12:383. [PubMed: 24175930]

80. Wells TNC, et al. Targeting the hypnozoite reservoir of Plasmodium vivax: the hidden obstacle to malaria elimination. Trends Parasitol. 2010; 26:145-151. [PubMed: 20133198] 


\section{Highlights}

- Persons with asymptomatic malaria are an important reservoir of transmission.

- Smear-positive asymptomatics are more infectious to mosquitoes than those with submicroscopic infection.

- Current efforts to eliminate the malaria reservoir often target those with submicroscopic malaria.

- The contribution of submicroscopic malaria to transmission needs further evaluation. 


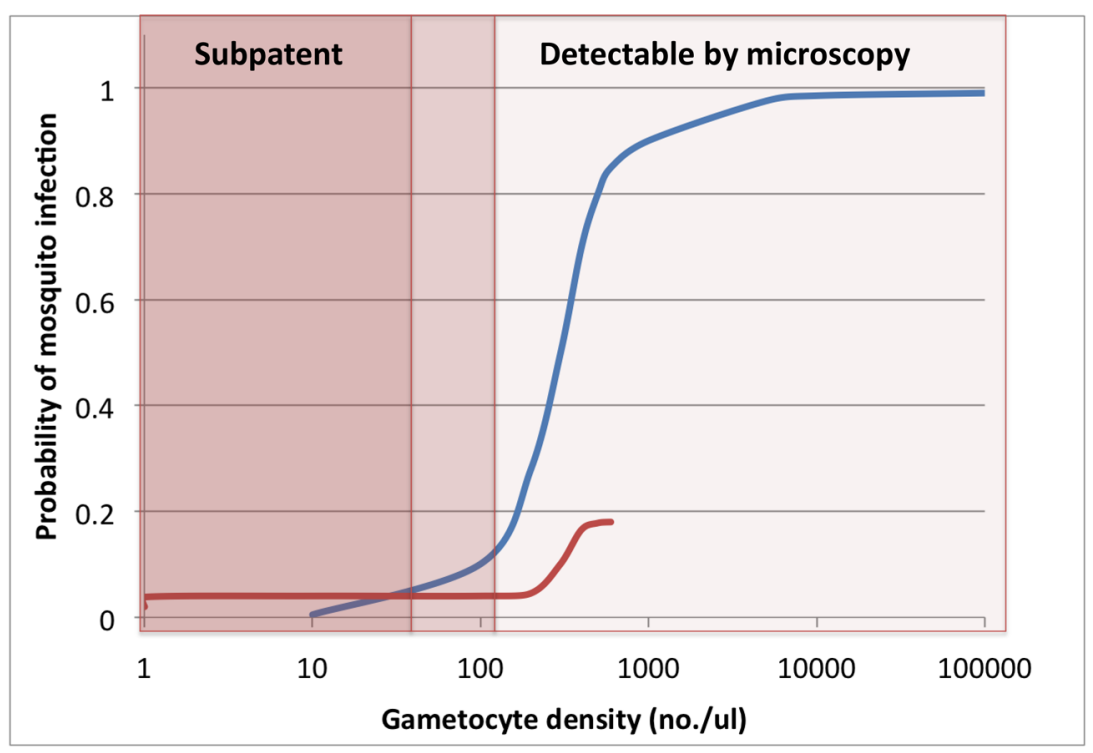

Figure 1. Submicroscopic gametocytemia and the mosquito infectivity threshold Mosquito feeding assays, whereby Anopheles mosquitoes are fed blood-stage parasites, either by direct skin feeding or via a membrane feeding apparatus, and subsequently dissected to look for malaria parasites, represent the standard way of assessing human-tomosquito infectious potential $[33,34,35,36]$. Depicted are approximate curves of human-tomosquito infectivity as a function of density of gametocytes in the circulating blood drawn from data depicting natural human malaria infections in West Africa (blue) $[39,40]$ and children post-treatment in Burkina Faso and Kenya (red) [41]. While the range of the older series is much greater, both demonstrate that the probability of mosquito infection is very low until gametocyte densities well within the range of parasitemia detectable by microscopy. 